\title{
Plantar Fasciopathy, General Concepts, Shock Wave Treatment and Other Additional Therapeutic Considerations
}

\author{
Osvaldo Valle Toledo ${ }^{1}$
}

\section{Abstract}

Plantar fasciopathy is the most common cause of heel pain. It is a primarily degenerative and mechanical overuse pathology. The plantar fascia fulfills important biomechanical functions in the foot, being its "windlass" mechanism, the most important function in this regard, allowing the foot to act as a single and efficient motor unit during gait. Its clinical and imaging diagnosis is fully defined, being Baxter's nerve entrapment neuropathy, its most significant differential diagnosis. The elongation exercises constitute the basic treatment, being the extracorporeal shock wave therapy of significant utility, amplified in its effects by the association with the referred therapeutic exercises.

Keywords: Plantar fasciitis, shock waves, fasciopathy.

\section{Introduction}

TThe plantar fascia plays a very significant role in the biomechanics of the foot, both in static and walking positions. It supports the longitudinal arch of the plantar arch supporting only $14 \%$ of the total weight load that the foot receives, it participates in the absorption and dissipation of the podal mechanical impacts and during walking, it becomes an essential part of the RIDING mechanism or "WINDLASS" mechanism of the foot: the plantar fascia tightens during the propulsion phase, after dorsiflexion of the orthotics, which in turn conditions the elevation of the longitudinal arch of the foot, with a subsequent shortening/compaction effect of the foot [1]. Thus, in the propulsive phase of gait (third rocker), the foot moves forward as a single, functionally efficient mechanical unit.

The triangular configuration of the plantar fascia favors the load-bearing functions it must fulfill [1]. The plantar fascia containts the plantar podal musculature.

\section{Etiology and Pathophysiology}

Plantar fasciitis (or better said fasciopathy or fasciosis) is a primarily degenerative and overuse condition, and it is not an acute inflammatory process. In its histopathology, there are no acute phase inflammatory cells, but granulatory tissue is found, myxoid degenera-tion, microtears and alteration of the collagen fibers in their linear spatial arrangement, together with changes in the type of structural collagen of the fascia, with substitution of the mechanically elastic collagen Type I, by the mechanically more rigid collagen Type III [2].

It is a clearly self-limited pathology, tending to spontaneous clinical resolution in a variable time in most cases [2]. About $70-80 \%$ of patients show spontaneous resolution within 9-12 months [2].

The vast majority of cases (90\%) resolve with conservative treatment within 12 months or sooner. 5-10\% will be absolutely recalcitrant or refractory to any form of conservative management and will ultimately require surgical management. The highest morbidity and recurrence rate occurs in the athlete population [2].

Patients with valgus flat feet, contracture or shortening of the gastrocnemius with second-ary limited tibiotalar dorsiflexion (diagnosis based on the Silfverskiöld test) and severe hallux valgus, are the most likely to suffer a fasciopathy [3]. Eighty three percent
${ }^{1}$ Department of Orthopedics and Traumatology, Ankle Foot Subspecialist, Ankle-Foot

Team, MEDS Clinic, Santiago de Chile.

Address of Correspondence

Dr. Osvaldo Valle Toledo, MD,

Department of Orthopedics and Traumatology, Ankle Foot Subspecialist, Ankle-Foot

Team, MEDS Clinic, Santiago de Chile.

E-mail: osvaldovalletoledo@yahoo.es

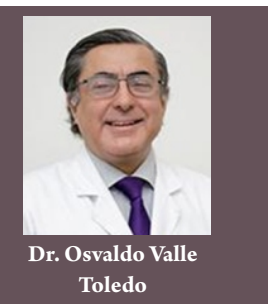

of patients with refractory or recalcitrant chronic plantar fasciopathy have limited tibiotalar dorsiflexion.

The main underlying biomechanical alteration can be summarized in that the increase of the tension of the gastrocnemius, causes in the final phase of the second rocker, a combination of moments of plantar flexion of the rearfoot with moments of dorsal flexion of the forefoot, which in turn conditions an increase of the passive longitudinal tension of the plantar fascia, that tries to oppose to the effect of flattening of the longitudinal arch generated by the increased tension of the gastrocnemius.

Talonavicular instability and spring ligament insufficiency, with consequent mechanical overload of the medial plantar fascia, have also been considered as biomechanical determinants of subsequent degenerative histological alteration of the medial plantar fascia [4].

The following have also been considered as intrinsic predisposing factors: cavus feet, limited tibiotalar dorsiflexion (less midfoot and forefoot dorsiflexion), greater ankle abduction, increased rearfoot adduction, greater degree of forefoot eversion, increased hip abduction, decreased coxofemoral and knee extension, women with narrow and long feet, among others [5].

Between $59 \%$ and $66 \%$ of patients with heel pain and between $67 \%$ and $89 \%$ of patients with plantar fasciopathy have a heel spur, but the spur is not the cause of the pain: most of them also have a spur in the contralateral

Submitted Date: 13 December 2021, Review Date: 17 December 2021, Accepted Date: 20 December 2021 \& Published: 31 December 2021

(C) 2021 by Journal of Regenerative Science | Available on www.jrsonweb.com | DOI:10.13107/jrs.2021.v01.i01.021

This is an open access journal, and articles are distributed under the terms of the Creative Commons Attribution-NonCommercial-ShareAlike 4.0 License (https://creativecommons.org/licenses/by-nc-sa/4.0/), which allows others to remix, tweak, and build upon the work non-commercially, as long as appropriate credit is given and the new creations are licensed under the identical terms. 
asymptomatic foot [6]. In any case, it should be considered that in $45-85 \%$ of patients with plantar fasciopathy, the presence of calcaneal spurs is verified. However, $65 \%$ of asymptomatic patients also present this anatomical finding $[3,6]$.

Anatomical studies show that the spur originates more frequently in the insertion of the flexor digitorum brevis of the toes and not in the insertional plantar fascia. It has also been found located, in a smaller percentage of cases, in relation to the insertions of the abductor hallux, abductor digiti minimi and plantar quadratus $[6,7,8,9]$. No correlation has been found between the different anatomical forms of calcaneal spurs with the presence or ab-sence of pain. Nowadays, it is considered only as a radiological finding [10].

However, a finding of a large or especially bulky calcaneal spur may eventually be related to an entrapment of Baxter's nerve [8].

It should also be considered that histologically, a calcaneal spur is a nucleus of mature lamellar bone associated with fibrocartilaginous proliferation, and covered by a highly vascularized and innervated connective tissue, which could play a role in the genesis of heel pain [8].

As additional information, it should be noted that no correlation has been found between the shape of the calcaneal spur and the presence or absence of clinically significant pain [8].

Associated systemic diseases, such as seronegative spondyloarthropathies, may be found in $15 \%$ of cases, and this etiology should always be considered when the presentation is symptomatically intense, simultaneous bipodal and in young patients $[11,12]$.

Fasciitis is also more frequently found in overweight patients (46-76\%) [6].

In summary, plantar fasciopathy has an etiopathogenesis conditioned by mechanical over-use, associated with an underlying genetic/degenerative condition: type I collagen, mechanically efficient, is replaced by type III collagen, of lower mechanical functionality (less elasticity).

\section{Epidemiology}

Plantar fasciitis is the most common cause of heel pain. Its exact incidence and prevalence are unknown, but it is estimated that in the
United States, this pathology motivates one million medical consultations per year. It accounts for $11-15 \%$ of all consultations for painful symptomatic foot pain requiring professional follow-up; it is also the cause of $8-10 \%$ of all injuries in joggers $[13,14]$. It is estimated that one in ten people will develop at least one episode of plantar fasciopathy in their lifetime, with $85 \%$ of these patients being working population, between 25 and 65 years of age $[13,14]$.

Two major epidemiological groups are recognized as having a higher incidence: obese women in middle age and young male athletes. In the latter group, most require medical treatment and the neurological etiology of pain is important: between $15 \%$ and $20 \%$ of heel pain in athletes is related to neural compression. Compressive neuropathy of the first branch of the lateral plantar nerve of the posterior tibial or Baxter's nerve is considered to be the main cause of chronic heel pain in this group of patients. In one third of these cases the presentation may be bilateral.

On the other hand, the prevalence of heel pain secondary to neurological causes in the general population is unknown. Some literature demonstrates prevalence rates in the jogging population as high as $22 \%$ [ 13 , 14]. Conditioning neuropathies include tarsal tunnel syndrome (posterior tibial nerve entrapment) and entrapment of the first branch of the lateral plantar nerve: Baxter's nerve. Compressive neuropathy of the first branch of the lateral plantar nerve or Baxter's nerve is considered the main cause of chronic heel pain in athletes $[13,14]$. In one third of these cases the presentation is bilateral $[13,14]$. An entrapment of Baxter's nerve can be suspected clinically, by a deficit of abduction of the fifth toe or an asymmetric pattern of abduction compared to the contralateral toe. It can also be detected by a Tinnel sign on the inner aspect of the heel, in the abductor hallucis area or very intense pain on palpation of the posteromedial tuberosity of the cal-caneus.

\section{Clinical and Imaging Evaluation}

The most significant characteristic of plantar fasciopathy pain is its initial sharpness with the first foot support (for example, morning pain when getting out of bed or when starting to walk after a process of prolonged sitting), this pain decreases as ambulation progresses.
On physical examination, there is pain on palpation focused on the posteromedial tuberosity of the calcaneus (insertional site) or in its course along the rest of the foot, especially in the longitudinal arch. It is usual for this pain to diminish in intensity as walking time is prolonged.

Ultrasound and magnetic nuclear resonance are useful methods in the imaging evaluation, estimating as a consensus, an average thickness of the usual normal fascia of up to $3.0 \mathrm{~mm}$ measured with both techniques and as a pathological condition thicknesses of 4.0 mm or more [15]. However, there are studies that question this diagnostic condition and place greater emphasis on the degree of elasticity of the plantar fascia as a primordial element in the diagnosis [15].

Elastosonography has proved to be a very useful tool in the quantitative evaluation of the elasticity of the plantar fascia as a diagnostic element [15]; it has also demonstrated a high correlation with clinical parameters of pain reduction and functional improvement with treatment [16].

MRI allows a differential diagnosis, especially with compressive neuropathies of Baxter's nerve (first branch of the lateral plantar nerve of the posterior tibial nerve), detecting edema, fatty infiltration and/or atrophy of the abductor digiti minimi muscle, as an indirect sign of this neural compressive phenomenon. Electromyography conduction velocity plays only a role in the differential diagnosis with carpal tunnel syndrome; but it lacks sensitivity and specificity for the detection of neural conduction disturbances at the level of Baxter's nerve. It could play an additional role in the diagnostic differentiation with sensitive peripheral polyneuropathies.

Shock Wave Treatment of Plantar Fasciitis and Other Therapeutic Considerations

In relation to this point, there are a number of statistical facts that can be reported. The success rates reported in numerous series of cases of plantar fasciitis treated with shock waves vary from $34 \%$ to $88 \%$ (considering different types of shock waves, varied protocols and multiple equipment) [17].

The symptomatic response to shock wave therapy is more effective in chronic plantar fasciopathy (more than 3 months of evolution since the onset of symptoms) than in acute plantar fasciopathy [18].

Level I clinical studies have demonstrated the 
usefulness of extracorporeal shock waves in the treatment of plantar fasciopathy $[3,19$, 20].

Since 2010, the American College of Foot and Ankle Surgeons has recommended extracor-poreal shock wave therapy as the treatment of choice for plantar fasciitis when the usual conservative treatment has failed and its role, in terms of reducing the incidence of surger-ies in the group of chronic evolution of this pathology [21].

Focused shock waves play a significant role in pain relief in patients with plantar fasciopathy, with greater effectiveness than radial pressure waves [22]. Radial pressure wave therapy has shown less clinical response than focal wave therapy, but when considering its lower cost, its effectiveness was greater (cost/effectiveness criterion) [23].

If analgesic response and remission of functional disability are compared between treat-ment with shock waves versus conventional physiotherapy, the response with both, at three months of evolution, is similar, but conventional physiotherapy consumes more time [24].

Local anesthesia has a negative influence on the results of pain relief when applied in conjunction with extracorporeal shock waves in the treatment of chronic plantar fasciitis [25]. In relation to the comparison between infiltration with local deposit corticosteroids and application of shock waves, it is clear that the pain relief response is faster with the first technique than with the second, but these results begin to be more favorable for shock waves after 3 months of follow-up. However, infiltration turns out to be more costeffective, and therefore, it is not a bad scheme to try it as a first therapeutic approach, to be followed by the application of shock waves. However, the application of corticosteroid infiltrations more than three times, before the application of shock waves, turns out to be a negative predictive factor with respect to their therapeutic success [17]. Radial pressure waves, according to evaluation in meta-analysis, seem to be more cost effective than fo-cused shock waves, but the isolated clinical outcome slightly favors the latter [23].

There is some evidence that, when comparing infiltrations with platelet-rich plasma versus extracorporeal shock waves in the treatment of chronic plantar fasciopathy, both treatments are useful, with a statistically significant advantage in terms of greater pain relief, favoring the treatment with plasma (prospective randomized study with serial follow-up between 2 and 24 weeks) [26]; the results of infiltration with platelet-rich plasma are improved and the procedure is performed in an ultrasound-guided manner $[3,26]$.

In a meta analysis study, it was found that there is moderate to low evidence of the useful-ness of dry needling for the relief of pain trigger points in symptomatic plantar fascia [27].

No comparative studies of this technique with the application of extracorporeal shock waves have been found in the literature to date.

A considerable decrease in the success rate of treatment with extracorporeal shock waves has been demonstrated in patients with gastrocnemius contracture or shortening [3]. Therefore, the use of eccentric triceps suralis exercises with additional focused plantar fascia stretching exercises is now considered to be possibly the conservative treatment of choice if a single management measure for chronic plantar fasciopathy is to be decided on [28].

The combination of shock waves and stretching/elongation of triceps suralis and plantar fascia was found to be more effective in the treatment of plantar fasciopathy in a series of articles reviewed between 2012 and 2017 than single conventional physiotherapy [29]. Combining shock waves with orthotic insoles appears to produce a greater decrease in pain in plantar fasciopathy than shock wave therapy alone [29].

Orthopedic insoles with anatomical adaptation to the plantar podal contour are more effec-tive in relieving the symptoms of plantar fasciopathy than enhancement heel cushions (silicone heel cushions with a soft center) [30]. "Taping” type bandages are only useful for short periods of time and therefore are not a recommendable measure [30], and night splints are poorly tolerated and are not a recommendable measure [30]. On the other hand, the combination of night splints or rocker shoes, plus the use of orthopedic insoles, is a more effective treatment than each management measure separately [30].

About $95 \%$ of patients with plantar fasciopathy will be pain-free, under conservative treatment, at 12 months; therefore, surgery should never be considered as an alternative before this period. Several studies have compared the application of focused shock waves with respect to percutaneous plantar fascia surgery, supporting the first therapy with respect to the second, both for its effectiveness and because patients can quickly resume their full work functions and in the case of athletes, they can maintain their training dynamics and sports practice in a continuous way [31]. At present, medial gemellar release is the treatment of choice as a single surgical procedure when a staged conservative treatment scheme has failed, and can be combined with partial insertional release of the plantar fascia.

Infiltrations with autologous blood, dextrose and botulinum toxin have shown nonpredictable effects, with benefits and response times of remission of symptomatology very variable [3].

In relation to the identification of prognostic factors of positive evolution under treatment, the presence of bone edema in the insertion of the plantar fascia in the calcaneus has been identified as a favorable prognostic factor for response to radial pressure waves, and the presence of edema in perifascial soft tissues as a favorable prognostic factor for infiltration with corticoids [32].

\section{Conclusions}

Plantar fasciopathy is a self-limited condition, with response to conservative treatment in $90 \%$ of patients between 9 and 12 months.

The limitation of tibiotalar dorsiflexion is the most important biomechanical risk factor for the development of plantar fasciopathy.

The conservative treatment of choice is eccentric calf exercises associated with plantar fascia stretching exercises.

Extracorporeal shock waves are a conservative treatment that should be implemented after failure of other nonsurgical measures and their combination with stretching or stretching exercises of triceps suralis and plantar fascia, is a real alternative treatment, validated from the statistical point of view. The use of focused waves should be technically preferred over radial pressure waves.

Surgery plays only an exceptional role in the treatment of plantar fasciopathy and should be considered only as the last link in a chain of 
stepwise treatments, only in the case of proven failure of well-implemented conservative measures.

Declaration of patient consent: The authors certify that they have obtained all appropriate patient consent forms. In the form, the patient has given his consent for his images and other clinical information to be reported in the Journal. The patient understands that his name and initials will not be published, and due efforts will be made to conceal his identity, but anonymity cannot be guaranteed.

Conflicts of Interest: Nil. Source of Support: None.

\section{References}

1. Rodríguez. Qué es la Fascia Plantar? 2015. Available from: https://lafisioterapia.net/que-es-la-fascia-plantar [Last accessed on 2021 Dec 12].

2. Buchanan BK, Kushner D. Plantar Fasciitis. Treasure Island, FL: StatPearls Publishing; 2021.

3. Monteagudo M, de Albornoz PM, Gutierrez B, Tabuenca J, Álvarez I. Plantar fasciopathy: A current concepts review. EFORT Open Rev 2018;3:485-93.

4. Pasapula C, Kiliyanpilakkil B, Khan DZ, Di Marco Barros R, Kim S, Ali $A M$, et al. Plantar fasciitis: Talonavicular instability/spring ligament failure as the driving force behind its histological pathogenesis. Foot (Edinb) 2021;46:101703.

5. Harutaichun P, Boonyong S, Pensri P. Differences in lower-extremity kinematics between the male military personnel with and without plantar fasciitis. Phys Ther Sport 2021;50:130-7.

6. Kirkpatrick J, Yassaie O, Mirjalili SA. The plantar calcaneal spur: A review of anatomy, histology, etiology and key associations. J Anat 2017;230:74351.

7. Li J, Muehleman C. Anatomic relationship of heel spur to surrounding soft tissues: Greater variability than previously reported. Clin Anat 2007;20:950-5.

8. Díaz-Llopis IV. Despejando dudas sobre la fascitis plantar. XXIX Congreso de la Sociedad Valenciana de Medicina Física y Rehabilitación. Slides Presentation. Available from: https://svmefr.com/wpcontent/uploads/2020/03/ISMAEL-DIAZ.pdf [Last accessed on 2021 Dec 12].

9. Forman WM, Green MA. The role of intrinsic musculature in the formation of inferior calcaneal exostoses. Clin Podiatr Med Surg 1990;7:217-23.

10. Acosta TB, Pérez YM, Tápanes SH, Cordero JE, Lottie AG, Aliaga B, et al. Bibliographic review. Rev Iberoamericana Fisiot Kinesiol 2008;11:2631.

11. Finkenstaedt T, Siriwanarangsun P, Statum S, Biswas R, Anderson KE, Bae WC, et al. The calcaneal crescent in patients with and without plantar fasciitis: An ankle MRI study. AJRAm J Roentgenol 2018;211:1075-82.

12. Arnold MJ, Moody AL. Common running injuries: Evaluation and management. Am Fam Physician 2018;97:510-6.

13. Cotchett M, Lennecke A, Medica VG, Whittaker GA, Bonanno DR. The association between pain catastrophising and kinesiophobia with pain and function in people with plantar heel pain. Foot (Edinb) 2017;32:8-14.

14. Tschopp M, Brunner F. Diseases and overuse injuries of the lower extremities in long distance runners. Z Rheumatol 2017;76:443-50.

15. Baur D, Schwabl C, Kremser C, Taljanovic MS, Widmann G, Sconfienza LM, et al. Shear wave elastography of the plantar fascia: Comparison between patients with plantar fasciitis and healthy control subjects. J Clin Med 2021;10:2351.

16. Schillizzi G, Alviti F, D'Ercole C, Elia D, Agostini F, Mangone M, et al. Evaluation of plantar fasciopathy shear wave elastography: A comparison between patients and healthy subjects. J Ultrasound 2021;24:417-22.

17. Yucel I, Ozturan KE, Demiraran Y, Degirmenci E, Kaynak G. Comparison of high-dose extracorporeal shockwave therapy and intralesional corticosteroid injection in the treatment of plantar fasciitis. $J$ Am Podiatr Med Assoc 2010;100:105-10.
18. Puttaswamaiah $R$, Chandran P. Degenerative plantar fasciitis: A review of current concepts. Foot 2007;17:3-9.

19. Buchbinder R, Ptasznik R, Gordon J, Buchanan J, Prabaharan V, Forbes $A$. Ultrasound-guided extracorporeal shock wave therapy for plantar fasciitis: A randomized controlled trial. JAMA 2002;288:1364-72.

20. Aqil A, Siddiqui MR, Solan M, Redfern DJ, Gulati V, Cobb JP. Extracorporeal shock wave therapy is effective in treating chronic plantar fasciitis: A meta-analysis of RCTs. Clin Orthop Relat Res 2013;471:364552.

21. Moya D, Ramón S, Schaden W, Wang CJ, GuiloffL, Cheng JH. The role of extracorporeal shockwave treatment in musculoskeletal disorders. J Bone Joint Surg Am 2018;100:251-63.

22. Sun J, Gao F, Wang Y, Sun W, Jiang B, Li Z. Extracorporeal shock wave therapy is effective in treating chronic plantar fasciitis: A meta-analysis of RCTs. Medicine (Baltimore) 2017;96:e6621.

23. Chang KV, Chen SY, Chen WS, Tu YK, Chien KL. Comparative effectiveness of focused shock wave therapy of different intensity levels and radial shock wave therapy for treating plantar fasciitis: A systematic review and network meta-analysis. Arch Phys Med Rehabil 2012;93:125968.

24. Greve JM, Grecco MV, Santos-Silva PR. Comparison of radial shockwaves and conventional physiotherapy for treating plantar fasciitis. Clinics (Sao Paulo) 2009;64:97-103.

25. Rompe JD, Meurer A, Nafe B, Hofmann A, Gerdesmeyer L. Repetitive low-energy shock wave application without local anesthesia is more efficient than repetitive low-energy shock wave application with local anesthesia in the treatment of chronic plantar fasciitis. $J$ Orthop Res 2005;23:931-41.

26. Haddad S, Yavari P, Mozafari S, Farzinnia S, Mohammadsharifi G. Platelet-rich plasma or extracorporeal shockwave therapy for plantar fasciitis. Int J Burns Trauma 2021;11:1-8.

27. Llurda-Almuzara L, Labata-Lezaun N, Meca-Rivera T, NavarroSantana MJ, Cleland JA, Fernández-de-Las-Peñas $C$, et al. Is dry needling effective for the management of plantar heel pain or plantar fasciitis? An updated systematic review and meta-analysis. Pain Med 2021;22:163041.

28. DiGiovanni BF, Nawoczenski DA, Lintal ME, Moore EA, Murray JC, Wilding GE, et al. Tissue-specific plantar fascia-stretching exercise enhances outcomes in patients with chronic heel pain. A prospective, randomized study. J Bone Joint Surg Am 2003;85:1270-7.

29. Avilés SG. Efectividad de las Ondas de Choque en la Fascitis Plantar. Revisión Sistemática. España: Alcalá la Real; 2017.

30. Schuitema D, Greve C, Postema K, Dekker R, Hijmans JM. Effectiveness of mechanical treatment for plantar fasciitis: A systematic review. J Sport Rehabil 2019;29:657-74.

31. Weil LS Jr., Roukis TS, Weil LS, BorrelliAH. Extracorporeal shock wave therapy for the treatment of chronic plantar fasciitis: Indications, protocol, intermediate results, and a comparison of results to fasciotomy. $J$ Foot Ankle Surg 2002;41:166-72.

32. Maier M, Steinborn M, Schmitz C, Stäbler A, Köhler S, Pfahler M, et al. Extracorporeal shock wave application for chronic plantar fasciitis associated with heel spurs: Prediction of outcome by magnetic resonance imaging. J Rheumatol 2000;27:2455-62. 


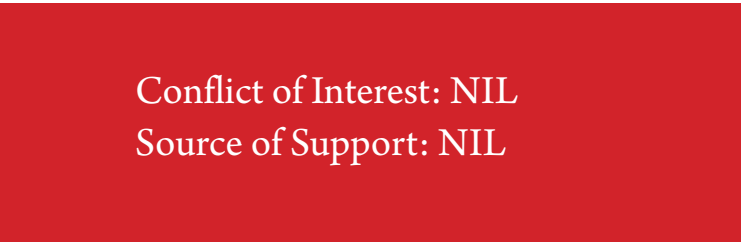

\section{How to Cite this Article}

Toledo OV | Plantar Fasciopathy, General Concepts, Shock Wave Treatment and Other Additional Therapeutic Consideration | Journal of Regenerative Science | December 2021; $1(1): 39-43$. 